\title{
EDITORIAL
}

\section{Defend the right ventricle with what you know}

\author{
M. Gomberg-Maitland
}

$\mathbf{R}$ ight ventricle failure in patients with pulmonary hypertension $(\mathrm{PH})$ has high mortality and is often unrecognised because the syndrome is less evident on exam than left heart failure. $\mathrm{PH}$ is an uncommon condition and management of patients with $\mathrm{PH}$ during critical illness is largely based on experience, as data from randomised clinical trials in this specific population do not exist. At the academic medical centres where relatively large numbers of $\mathrm{PH}$ patients are treated, the specialists and the intensivists who work with them share a common experience. Patients with decompensated right ventricle function and cardiogenic shock can look surprisingly stable, but reversing the shock or managing these brittle $\mathrm{PH}$ patients through an elective or emergent surgical procedure involves a delicate balance. Each intervention is determined by a mixture of physician experience, inference from physiology and by local practice norms, not randomised investigations.

In the current issue of the European Respiratory Journal, French clinician-scientists provide descriptive results of the largest database collected to date on patients experiencing acute right heart failure requiring admission to an intensive care unit [1] and patients requiring surgical procedures [2] at their internationally recognised centre. There have been few publications regarding the management of and outcomes from acute decompensated right heart failure or the peri-operative management of patients with $\mathrm{PH}$. This prompted investigators in Paris to report their collective experience in these areas.

The readers of this journal are familiar with pulmonary arterial hypertension (PAH), a fatal group of diseases characterised by an elevated pulmonary vascular resistance with a normal pulmonary artery wedge pressure in the absence of chronic lung disease/hypoxia, chronic thromboembolic disease (CTEPH), or left heart disease. PAH is characterised by pulmonary vasoconstriction, endothelial dysfunction, excessive smooth muscle cell proliferation and in situ thrombosis [36]. The consequence of this process is right ventricular ischaemia, remodelling, dilatation, dysfunction and eventually failure $[7,8]$. The decreased right ventricular cardiac output in conjunction with the altered septal shift impairing left ventricular filling, ultimately decreases left ventricular cardiac output. This then amplifies right ventricular ischaemia [8, 9]. The right ventricle adapts but as the ventricular dysfunction progresses with increasing pulmonary vascular resistance (PVR), it is unable to respond to situations (tachycardia,

Section of Cardiology, Dept of Medicine, University of Chicago, Chicago, IL, USA.

CORRESPONDENCE: M. Gomberg-Maitland, University of Chicago Hospitals, 5841 S Maryland Ave, MC 5403, Room \# L08, Chicago, IL 60637, USA. E-mail: mgomberg@medicine.bsd.uchicago.edu hypotension and hypoxemia) that require acute augmentation of cardiac output to maintain normal function. Presently, despite the heterogeneity of $\mathrm{PAH}$, due to the clinical and pathological similarities, the sub-conditions are treated by a unified approach. Current therapeutic interventions are based on each clinician's experience with right ventricle failure and animal models of right ventricle failure. The main principle is to maintain sufficient aortic and coronary perfusion pressure to sustain cardiac output (tissue perfusion) and tissue oxygenation [10-13]. Animal models support the use of dobutamine and norepinephrine alone or in combination $[14,15]$.

"Prognostic factors of acute heart failure in patients with pulmonary arterial hypertension," by SZTRYMF et al. evaluated consecutive PAH patients admitted to the intensive care unit from November 2005 to April 2007 [1]. All patients enrolled had been diagnosed to have had acute deterioration within 1 week of admission and required catecholamine treatment for cardiogenic shock. Despite aggressive care, the patients had a high in-hospital mortality rate $(41 \%)$, which is similar to the poor prognosis of cardiogenic shock due to left ventricular ischaemia [16]. These PH patients typically avoided severe heart failure requiring admission for at least $6 \mathrm{yrs}$ after diagnosis. At the last visit prior to hospitalisation, the cohort described had advanced disease; they were predominantly functional class III and IV with severe haemodynamics at right heart catheterisation and had already been prescribed a combination of background $\mathrm{PAH}$ therapies. These patient factors did not affect overall survival, only the presence of an infection worsened prognosis. Patients discharged had both a high readmission rate $(29 \%)$ and a high mortality rate $(22 \%)$ at 3 months.

Treatment plans were not structured and were guided by clinical measurement and individual physician experience, but the institutional custom was to administer intravenous dobutamine (at rates up to $20 \mu \mathrm{g} \cdot \mathrm{kg}^{-1} \cdot \mathrm{min}^{-1}$ ) as the first line vasoactive agent. If the maximum dose was reached without achieving adequate systemic blood pressure and objective signs of tissue perfusion, norepinephrine was added. Clinical endpoints consistent with shock and right ventricle failure predicted a worse prognosis. Specifically, adverse events increased if patients on admission to the intensive care unit required a larger dose of oral diuretic (furosemide 250 (60$1,500)$ versus $170(40-1,000) \mathrm{mg} \cdot$ day $^{-1}$, had worse renal function as measured by serum creatinine (112 (42-144) versus 95 (85151) $\left.\mu \mathrm{mol} \cdot \mathrm{L}^{-1}\right)$ or required a progressive increase in dobutamine dose $\left(5-20 \mu \mathrm{g} \cdot \mathrm{kg}^{-1} \cdot \mathrm{min}^{-1}\right)$. Understanding that the study is non-randomised and underpowered to evaluate this outcome, despite the addition of emergent PAH medications, 
clinical prognosis did not improve. In addition, this decision was based on the discretion of each physician without clear guidelines directing their use. The physicians avoided the use of mechanical ventilatory support and bronchoscopic confirmation of infection to limit procedural complications and cardiovascular compromise. They also used antibiotics prior to confirmatory cultures and managed fluids sparingly.

Attentive support of the right ventricle is also needed by patients who require operative procedures. The need to optimise right ventricle function to prevent right ventricle failure pre, intra, and post-operatively is underappreciated. Obstetric and paediatric operative experience dominates the limited literature [17-19]. Preventive measures include the use of pulmonary vasodilator therapies, restriction of intravenous fluid administration and maintenance of systemic pressure. In the second article in this issue of the European Respiratory Journal, PRICE et al. [2] report the acute results and 1-yr followup of consecutive PAH (WHO category 1) and inoperable CTEPH patients (WHO Category IV) who underwent general or regional anaesthesia for non-cardiothoracic, non-obstetric surgery. The authors provide detailed reports on a cohort of patients, providing details of their anaesthesia (including nitrous oxide), mechanical ventilation, oxygen requirements, and peak end-expiratory carbon monoxide during surgery and report the peri-operative (28 days) complications and mortality.

Unlike SzTRYMF et al. [1], the patients in this study had mildmoderate $\mathrm{PH}$, good functional class (I and $\mathrm{II}=75 \%$ ), a mild-moderate elevation in pulmonary pressure (mean= $43 \pm 12 \mathrm{mmHg}$ ) and a normal cardiac performance (cardiac index $=3.25 \pm 0.68 \mathrm{~L} \cdot \mathrm{min}^{-1} \cdot \mathrm{m}^{-2}$ ). Despite well-compensated $\mathrm{PH}$, nearly one-third of patients $(29 \%)$ had peri-operative complications (most within $48 \mathrm{~h}$ ) and $7 \%$ had peri-operative mortality. Major surgery defined as major laparoscopy, hysterectomy, major orthopaedic surgery and mastectomy (not typically included), resulted in a 13\% mortality (two out of 16). No deaths occurred with minor surgery. General anaesthesia patients received opioids and propofol for anaesthesia induction, sevoflurane (10 out of 14) or desflurane (two out of 14) for maintenance and nitric oxide (four out of 14). Regional anaesthesia consisted of neuraxial blockade with sufentanil, bupivicaine and $2 \%$ lidocaine epidural top offs (11 out of 28) and three regional blocks combined with sedation. Patients who had general anaesthesia had less severe baseline haemodynamics and were less likely to be treated with $\mathrm{PH}$ specific medication. This may represent a selection bias with higher functioning patients having general analgesia, falsely improving general anaesthesia outcomes. Also most were emergency or major procedures confounding the comparison between anaesthetic techniques.

Some results contradicted previous management and should change current practice. For example, patients tolerated regional anaesthesia well despite fears of bolus administration of anaesthetic leading to hypotension. If patients survived the hospitalisation (26 out of 28), they returned to baseline clinical status by 3-6 months even if they had significant complications (six out of 26). As expected, emergency procedures carried a significantly higher risk of death and complications versus elective procedures (50\% (two out of four) and $100 \%$ (four out of four) versus $0 \%$ and $17 \%$ (four out of 24) respectively). Longer procedures and those requiring haemodynamic monitoring also increased complication rates. Limited exercise capacity and functional class appeared to predict outcomes similar to peri-operative risk assessment in cardiac patients having non-cardiac surgery $(<4$ metabolic equivalents (METs)) [20]. Perhaps not surprisingly, the stresses endured during exercise testing best simulated operative stress compared with resting cardiac catheterisation haemodynamics in patients with normal cardiac output. Thus exercise testing should be a primary recommendation for risk stratification. The predictive cut-points obtained from our experience using metabolic equivalents obtained from a modified Naughton-Balke protocol stress test can be utilised and validated for this purpose ( $<3$ METs, 3-6 METs and $>6$ METs) [21].

These collective experiences reiterated the challenges faced by the clinician in the care of $\mathrm{PH}$ patients. This difficult patient population dictates that care should be done in centres with experienced $\mathrm{PH}$ clinicians. Although institution-specific infrastructure and operations also influenced outcome, these data provide the first necessary information to begin developing clinical care guidelines and acute care clinical trials. For both the acute right heart failure and the peri-operative admission, these investigators prioritised careful fluid management. Unlike the treatment of right ventricle infarction that requires fluid resuscitation to support the acute dysfunction [22], right ventricle adaptation in $\mathrm{PAH}$ is such that this therapeutic approach would further stress the right ventricle. The authors mentioned the importance but did not report continuous central venous pressure monitoring. Our institutional experience is that this is a helpful method to measure right ventricle recovery and fluid balance. Perhaps setting haemodynamic goals such as maintenance of even to net negative fluid balance or central venous pressure $\pm 3 \mathrm{mmHg}$ from baseline would prove effective. To improve coronary perfusion and lessen ischaemia, another goal might be to maintain the mean arterial blood pressure $10 \mathrm{mmHg}$ above the mean pulmonary artery pressure. Although arbitrary, setting objective benchmarks like these would provide the basis for multi-centre prospective studies.

The authors are commended for their contributions to our understanding of the clinical management and outcomes of these complex patients. Novel therapeutics and more aggressive approaches with assist devices and early transplant in acute care plus the use of this support during highrisk procedures, may be beneficial in the future and warrant consideration. Formal studies of optimal management of right ventricle failure or cardiogenic shock in $\mathrm{PH}$, similar to the early days of the Acute Respiratory Disease Syndrome network or left heart failure, are needed. This noble goal can only be accomplished through international collaboration. The key to progress is not only in the recognition of right ventricle failure in $\mathrm{PAH}$ but also a humble appreciation for our ignorance in treating acute right ventricle failure.

\section{STATEMENT OF INTEREST}

A statement of interest for M. Gomberg-Maitland can be found at www.erj.ersjournals.com $/ \mathrm{misc} /$ statements.dtl 


\section{REFERENCES}

1 Sztrymf B, Souza R, Bertoletti L, et al. Prognostic factors of acute heart failure in patients with pulmonary arterial hypertension. Eur Respir J 2010; 35: 1286-1293.

2 Price LC, Montani D, Jaïs X, et al. Noncardiothoracic nonobstetric surgery in mild-to-moderate pulmonary hypertension. Eur Respir J 2010; 35: 1294-1302.

3 Chin KM, Rubin LJ. Pulmonary arterial hypertension. J Am Coll Cardiol 2008; 51: 1527-1538.

4 Tuder R, Chacon M, Alger L, et al. Expression of angiogenesisrelated molecules in plexiform lesions in severe pulmonary hypertension: evidence for a process of disordered angiogenesis. J Pathol 2001; 195: 367-374.

5 Wagenvoort C, Mulder G. Thrombotic lesions in primary plexogenic arteriopathy. Chest 1993; 103: 844-849.

6 Wagenvoort C, Wagenvoort N. Pathology of pulmonary hypertension. 2nd edn. New York, John Wiley and Sons, 1977.

7 Bogaard HJ, Abe K, Vonk Noordegraaf A, et al. The right ventricle under pressure: cellular and molecular mechanisms of right-heart failure in pulmonary hypertension. Chest 2009; 135: 794-804.

8 McLaughlin VV, Archer SL, Badesch DB, et al. ACCF/AHA 2009 expert consensus document on pulmonary hypertension: a report of the American College of Cardiology Foundation Task Force on Expert Consensus Documents and the American Heart Association: developed in collaboration with the American College of Chest Physicians, American Thoracic Society, Inc., and the Pulmonary Hypertension Association. Circulation 2009; 119: 2250-2294.

9 Chemla D, Castelain V, Herve P, et al. Haemodynamic evaluation of pulmonary hypertension. Eur Respir J 2002; 20: 1314-1331.

10 Piazza G, Goldhaber SZ. The acutely decompensated right ventricle: pathways for diagnosis and management. Chest 2005; 128: 1836-1852.

11 van Wolferen SA, Marcus JT, Westerhof N, et al. Right coronary artery flow impairment in patients with pulmonary hypertension. Eur Heart J 2008; 29: 120-127.
12 Vlhakes G, Turley K, Hoffman J. The pathophysiology of failure in acute right ventricular hypertension: hemodynamic and biochemical correlation. Circulation 1981; 63: 87-95.

13 Zamanian RT, Haddad F, Doyle RL, et al. Management strategies for patients with pulmonary hypertension in the intensive care unit. Crit Care Med 2007; 35: 2037-2050.

14 Kerbaul F, Rondelet B, Demester JP, et al. Effects of levosimendan versus dobutamine on pressure load-induced right ventricular failure. Crit Care Med 2006; 34: 2814-2819.

15 Kerbaul F, Rondelet B, Motte S, et al. Effects of norepinephrine and dobutamine on pressure load-induced right ventricular failure. Crit Care Med 2004; 32: 1035-1040.

16 Hochman JS, Sleeper LA, Webb JG, et al. Early revascularization in acute myocardial infarction complicated by cardiogenic shock. SHOCK Investigators. Should We Emergently Revascularize Occluded Coronaries for Cardiogenic Shock. N Engl J Med 1999; 341: 625-634.

17 Bedard E, Dimopoulos K, Gatzoulis MA. Has there been any progress made on pregnancy outcomes among women with pulmonary arterial hypertension? Eur Heart J 2009; 30: 256-265.

18 Minai OA, Venkateshiah SB, Arroliga AC. Surgical intervention in patients with moderate to severe pulmonary arterial hypertension. Conn Med 2006; 70: 239-243.

19 Lai HC, Wang KY, Lee WL, et al. Severe pulmonary hypertension complicates postoperative outcome of non-cardiac surgery. $\mathrm{Br} J$ Anaesth 2007; 99: 184-190.

20 Gregoratos G. Current guideline-based preoperative evaluation provides the best management of patients undergoing noncardiac surgery. Circulation 2008; 117: 3134-3144; discussion 3134.

21 Shah SJ, Thenappan T, Rich S, et al. Value of exercise treadmill testing in the risk stratification of patients with pulmonary hypertension. Circ Heart Fail 2009; 2: 278-286.

22 O'Rourke RA, Dell'Italia LJ. Diagnosis and management of right ventricular myocardial infarction. Curr Probl Cardiol 2004; 29: 6-47. 